\title{
LOS «DIPUTADOS MÉDICOS»: CLÍNICA Y POLÍTICA EN LA DISPUTA POR LOS RECURSOS PÚBLICOS EN BUENOS AIRES (1906-1917)
}

\section{Pablo Souza y Diego Hurtado}

\author{
Universidad Nacional de San Martín - CONICET
}

\begin{abstract}
RESUMEN
En junio 1906 el diputado conservador y médico Eliseo Cantón presentó en el Parlamento argentino el proyecto de «un gran hospital policlínico», que ocuparía cuatro manzanas, se ubicaría frente a la Facultad de Medicina de la Universidad de Buenos Aires y estaría integrado por 20 institutos con capacidad de 80 camas cada uno. La disputa alrededor de este proyecto faraónico, que se extendió hasta 1917, puso en el centro de la escena a un grupo de «diputados médicos» que utilizaron la tribuna política como espacio para la definición de aspectos centrales del dispositivo hospitalario. La polémica desbordó el Parlamento y alcanzó la esfera pública. La fastuosidad del proyecto en vísperas de los festejos del Centenario o la opulencia de una Buenos Aires que aspiraba a ser París fueron asociadas en el plano simbólico a los progresos de la medicina local y a sus aspiraciones a un lugar en la arena médica internacional. El caso analizado ayuda a comprender las modalidades en que una controversia esencialmente política, que desplegó argumentos tanto económicos, como éticos y cognitivos, intervino en el proceso de construcción del lugar socioprofesional de la práctica médica hospitalaria.
\end{abstract}

PALABRAS CLAVE: Eliseo Cantón. Enrique Dickman. Círculo Médico Argentino. Medicina clínica. Argentina.

\section{«MEDICAL DEPUTIES»: CLINICS AND POLITICS IN THE DISPUTE FOR PUBLIC RESOURCES IN BUENOS AIRES (1906-1917)}

\begin{abstract}
In June, 1906, the conservative deputy and doctor Eliseo Cantón submitted in the Argentinian Parliament the project of a «big polyclinical hospital», which would take up four blocks, would be located in front of the Faculty of Medicine of the University of Buenos Aires and would be made up by 20 institutes with a capacity for 80 beds each. The dispute over this huge project, which lasted until 1917, placed in the middle of the scene, a group of "medical deputies" who used the political platform as a space to define the main aspects of the hospital system. The controversy went beyond the Parliament reaching the public sphere. The lavishness of the project on the eve of the Centenary public festivities or the opulence of a Buenos Aires which aspired to be like Paris
\end{abstract}


were related, in a symbolic view, to the progress of local medicine and its aspirations to be included in the international medical arena. The analysed case helps understand how an essentially political controversy - which led to economical, ethical and cognitive discussions - intervened in the process of bulding up a socio-professional space of the hospital medical practice.

KEY WORDS: Eliseo Cantón. Enrique Dickman. Argentinian Medical Group. Clinical medicine. Argentina.

\section{INTRODUCCIÓN}

En junio 1906 el diputado conservador y médico Eliseo Cantón presentó en la Cámara de Diputados de la República Argentina el proyecto de «un gran hospital policlínico». La propuesta derivó en la ley 6026, promulgada en la ciudad de Buenos Aires en julio de 1908, por la cual se autorizaba a dar curso a uno de los proyectos científicos y médicos mas importantes de América Latina en su género, el Policlínico José de San Martín, que ocuparía cuatro manzanas, se ubicaría frente a la Facultad de Ciencias Médicas de la Universidad de Buenos Aires (UBA) y estaría destinado a reemplazar al antiguo Hospital de Clínicas de la ciudad de Buenos Aires, en funcionamiento desde 1883. El nuevo policlínico estaría integrado por 20 institutos con capacidad para 80 camas cada uno y su costo se estimaba en 15 millones de pesos. Este proyecto fue duramente cuestionado por el médico y diputado socialista Enrique Dickman, dando lugar a un importante cruce de cosmovisiones médicas y científicas que tuvo como epicentro el parlamento argentino.

La disputa alrededor de este proyecto faraónico y de la ley que buscó dar el marco legal a su materialización se extendió de manera intermitente hasta 1917 y puso en el centro de la escena a un grupo de «diputados médicos» que utilizaron la tribuna política como espacio para dirimir representaciones médicas divergentes. Desde la perspectiva de la historia social de la medicina, el presente artículo enfoca estos eventos como el estudio de una controversia que, si bien tuvo como escenario el parlamento, se concentró en cuestiones cruciales para la práctica clínica de comienzos del siglo veinte. Este cruce de la retórica política y la esfera médica, por varios motivos, se presenta como un caso especialmente apto para la exploración de dos cosmovisiones médicas en pugna y sus correlaciones con el contexto político y con algunos elementos centrales del imaginario social vigente sobre las prácticas hospitalarias. Mientras que la propia lógica del debate puso en la mira cuestiones centrales del dispositivo hospitalario, los registros propios de la retórica parlamentaria amplificaron aquellos significados no estabilizados dentro la co- 
munidad médica, al punto de que la polémica desbordó el parlamento y alcanzó la esfera pública.

Para contextualizar este debate, primero estableceremos un paralelismo entre dos principios legales: la mencionada ley 6026 de 1908, que desencadenó el conflicto que nos interesa analizar, y el decreto-ley 1284 de 1883, por el cual se posibilita la cesión del Hospital de Clínicas a la Facultad de Ciencias Médicas de la UBA. Esto nos permitirá presentar algunos materiales históricos — conceptuales y empíricos - e inscribir los sucesos desencadenados alrededor de la ley 6026 en un contexto socio-profesional de mayor amplitud. Seguidamente, presentaremos algunos datos biográficos relevantes de los diputados médicos que intervienen en la contienda político-científica y, en estrecha relación, el itinerario institucional de la ley en la Cámara de Diputados entre su primera presentación como proyecto en 1906 y su derogación en 1917. Finalmente, concentraremos la atención en la confrontación de los saberes clínicos sostenidos por ambos grupos contendientes, en especial en las distintas maneras de concebir la relación entre el sistema hospitalario y la salud de los habitantes de la ciudad.

\section{Marcos Legales Y CONFIGURACIÓN POLÍTICA DE LA MEDICINA CLÍNICA PORTEÑA A FINES DE SIGLO DIECINUEVE}

La ley 6096 de 1908 propuesta por el diputado Eliseo Cantón, representante de los sectores más tradicionales del cuerpo médico docente, no es la primera de importancia en materia de nosocomios que se debatió en la Cámara de Diputados. Ella remite al decreto-ley 1284 de agosto de 1883, que establece la cesión del Hospital de Clínicas a la Facultad de Ciencias Médicas de la UBA. Tres motivos fundamentan la comparación. Primero, ambas leyes son hitos importantes en la trayectoria de una misma visión del mundo médico. La medicina clínica reconoce en la primera fecha su momento de cristalización en el medio profesional y, en la segunda, un punto de inflexión o rediscusión. Las necesidades hospitalarias y sanitarias esgrimidas por ambos contendientes son similares a las razones que el poder ejecutivo expuso en el decreto por el que ordenó el traspaso del Hospital de Clínicas a la Facultad de Ciencias Médicas. Segundo, ambas leyes surgen en medio de fuertes pujas entre distintos grupos corporativos existentes en el seno de aquella facultad. Tercero, en paralelo a estas coincidencias, ambas leyes remiten a coyunturas históricas distintas - aunque causalmente vinculadas - de la profesión médica.

En primer lugar, digamos que la ley 1284 de cesión del Hospital de Buenos Aires a la Facultad de Ciencias Médicas en 1883 da origen a un Hospital 
Clínico u Hospital Escuela, celebrado como uno de los primeros de América latina. Esta ley es crucial en la historia de la medicina local, pues contribuyó a formar un dispositivo hospitalario complejo y fue espacio, al mismo tiempo, de formación médica y de atención. También es un hecho relevante el que comenzara a funcionar en 1880, durante los combates de Barracas, El Corral y Puente Alsina. Estos combates tuvieron lugar en la ciudad de Buenos Aires, en el marco del conflicto entre la provincia de Buenos Aires y el floreciente estado nacional. El motivo del conflicto fue la sucesión presidencial que debía darse en el año 1880. El resultado de las elecciones que habían designado presidente al general Julio A. Roca fue desconocido por Carlos Tejedor, gobernador de la provincia y segundo gran aspirante a la presidencia en aquellas elecciones. En dichos combates se enfrentaron las milicias provinciales y el ejército nacional solidario a Roca. El Hospital de Buenos Aires, construido por iniciativa de Carlos Tejedor a pedido del cuerpo médico en 1878, había sido terminado pocas semanas antes del conflicto y sirvió como centro de atención durante estos enfrentamientos ${ }^{1}$.

Así, la ley 1284 representa un momento de cristalización de la medicina clínica. A grandes rasgos, esta ley planteó la condición de posibilidad para la fusión, en el seno del hospital, de prácticas de trabajo y de un conjunto de saberes o estilos de pensamiento atravesados por el principio de «restitución de la normalidad» a los cuerpos de los pacientes ${ }^{2}$. Esta ley ayudó a la consolidación - a nivel del pensamiento profesional - de un haz de significados médico-clínicos señalados como clásicos en los estudios históricos de esta cosmovisión médica ${ }^{3}$. En efecto, surgen el Hospital y, en su interior, el estudio de las patologías a cargo del apóstol de la ciencia médica —el médico clínico-, realizado sobre el libro palpitante —el paciente hospitalario-

1 Cf. LobAto, M.Z. (2000), Estado gobierno y política en el régimen conservador. En LobAto M.Z. (comp.), Nueva Historia Argentina. El progreso, la modernización y sus límites (1880-1916), Buenos Aires, Editorial Sudamericana, p. 182; Cf. BontanA, N. (1977), El orden conservador, Buenos Aires, Hyspamerica, pp. 32-33; Cf. PÉrgola, F. y SANGuinetTi, F. (2000), Historia del Hospital de Clínicas, Buenos Aires, Ediciones Argentinas, p. 45.

2 SouZA, P.A. (2005), Formación histórica de un partido de la ciencia en la medicina argentina, 1875-1890. Tesis de Maestría, posgrado en Política sobre Ciencia Tecnología y Sociedad, Universidad de Buenos Aires, p. 173.

3 ACKerkneCht, E. (1967), Medicine at the Paris Hospital, 1794-1848, Baltimore, The Johns Hopkins Press, Capítulo 2, pp. 15-25. Puede verse también LESCH, J.E. (1988), The Paris Academy of Medicine and Experimental Science, 1820-1848. En ColEMAN, W. y Holmes, F.L. (1988), The Investigative Enterprise. Experimental Physiology in NineteenthCentury Medicine, California, University California Press, pp. 100-138, p. 101. 
inscrito en la cama del hospital. Este cuadro de situación propio de la profesión médica en los inicios de la década de 1880 se puede apreciar con claridad en los testimonios de quienes se desempeñaron en el Hospital de Clínicas durante sus primeros años, entre ellos, el joven médico Francisco Cobos.

El testimonio de Cobos es clave a la hora de apreciar algunos rasgos de una experiencia clínica local, en especial, el cuadro de situación interno dentro del nosocomio durante sus primeros años de enseñanza oficial. El flamante doctor dice a fines de $1885-\mathrm{a}$ un año y medio de funcionamiento formal del nosocomio- que:

[...] la experiencia ha demostrado a la generación presente, que la clínica es el objeto primordial de los estudios en las escuelas de medicina. Dedicado a la Clínica Médica durante mi internado en el Hospital de Clínicas, me he impuesto de sus necesidades e insuficiencias, y me ha parecido que podíamos dar un paso mas en pro de la enseñanza sin esperar en esto, como en los demás casos, el llamado de otros países, por adelantados que sean, si la innovación es útil, el ensayo es fácil y su realización ni costosa ni difícil ${ }^{4}$.

Por su parte, la ley 6026 ilustrará un momento de inflexión de esta cosmovisión médica. En el juego de argumentaciones y contra-argumentaciones que Cantón y Dickman desplegaron en torno al Policlínico José de San Martín, como se verá, aparecieron en un primer plano las debilidades que presentaban los distintos modelos de nosocomios para el sistema hospitalario de la ciudad. Se debatieron las distintas interpretaciones vigentes acerca del papel del hospital central y, también, la cantidad de camas necesarias para una población como la de la ciudad de Buenos Aires. Se discutió sobre el espacio ideal para un hospital, y sobre la cantidad de metros cuadrados por cama necesarios para que el «apóstol de la medicina» y el «libro palpitante» concretaran adecuadamente el ritual de la intervención. Se llegó incluso a litigar sobre la aceptación que poseían los hospitales entre los habitantes de Buenos Aires, pero en ningún momento surgió el tema de su centralidad en la vida del estudiante de medicina: en el sinuoso duelo verbal la utilidad del nosocomio para la formación del profesional médico. A inicios del siglo veinte, tal tópico estaba ampliamente consensuado dentro del cuerpo médico.

En segundo lugar, la similitud de las cosmovisiones debatidas en ambas leyes no debe opacar la existencia de contextos profesionales distintos. ¿Qué es lo que diferencia los momentos históricos en que lo que se inscribe cada

4 Anales del Círculo Médico Argentino (1886), Tomo 9, Buenos Aires, p. 81. 
ley? El decreto ley 1284 expresa un momento en que los conflictos entre distintos actores de la escuela médica no trascienden la unidad de dicha escuela. A pesar de las tensiones entre los actores corporativos, la escuela médica en su conjunto coincidió en la necesidad de trazar la geografía médica local, y avaló la necesidad de fundar un hospital escuela como uno de sus espacios primordiales.

Como podrá apreciarse a lo largo de estas páginas, la ley 6026 se inscribe en un momento de crisis y de replanteos en la profesión médica local ${ }^{5}$. Si a inicios de la década de 1880 los conflictos internos no obstaculizaron la aparición del Hospital de Clínicas, tres décadas mas tarde dichos conflictos llegaron al punto de la denuncia política explícita en la Cámara de Diputados de la Nación, dando por resultado el pedido de derogación de la ley 6026, que mandaba a construir un nuevo «hospital central» para la escuela. El conflicto entre Cantón y Dickman es isomorfo a un rasgo de la profesión en su conjunto vigente por estos mismos años, como es su crecimiento conflictivo y no ajeno a fuertes polémicas internas que llegaron a poner en cuestión temas de relevancia en la vida de la profesión ${ }^{6}$.

En tercer lugar, ¿qué grupos de la escuela médica protagonizaron las disputas alrededor de las leyes que son objeto de nuestra comparación? Como señalamos, ambas leyes no son ajenas a conflictos corporativos en el seno profesional. Antes bien, son una expresión de los mismos. González Leandri ha sostenido que el proceso de profesionalización de la medicina porteña durante la segunda mitad del siglo XIX generó tensiones y pujas entre distintos actores corporativos, motivadas por la búsqueda de un espacio en el medio profesional que asegurara su participación, tanto en la definición de las relaciones de poder dentro de los espacios institucionales, como en materia de producción intelectual. En este sentido, este trabajo se ha centrado en las relaciones sostenidas entre dos «corporaciones» de distinto tamaño y peso político. Por un lado, el cuerpo docente que dirige la vida institucional de la Facultad de Ciencias Médicas de la UBA y, por otro lado, los grupos críticos con el cuerpo docente, tales como estudiantes avanzados, graduados recientes, y médicos experimentados que buscaban hacerse un lugar dentro de la institución ${ }^{7}$.

5 Belmartino, S., Bloch, C., Persello, A.V., Carnino, M.I. (1988), Corporación Médica y Poder en Salud. Argentina, 1920-1940, Buenos Aires, Oficina Panamericana de la Salud, p. 15.

6 Ibíd.

7 Un estudio detallado de los actores que componen la profesión médica durante la segunda mitad del siglo XIX, puede verse en GONZÁLEZ LEANDRI, R. (2000), Curar, persuadir, 
Se ha referido en otro lugar que el proyecto de cesión del Hospital de Clínicas desde su aparición en 1878 hasta su efectiva entrega a la Facultad de Ciencias Médicas en 1883 creció en medio de los conflictos entre ambas corporaciones ${ }^{8}$. El surgimiento del Círculo Médico Argentino (CMA) en 1875 como organización gremial y científica que representó a los sectores disidentes marcó un punto de tensión en la disputa de modelos profesionales ${ }^{9}$. El CMA criticó con dureza el tipo de medicina practicado hasta entonces, centrado en la atención privada o en la «satisfacción del lucro personal». En su lugar, promovió la decidida adopción de la medicina clínica como eje formativo de la escuela médica, de moda en las principales capitales europeas desde fines del siglo dieciocho y principios del siglo diecinueve. El período 18801883 está poblado de conflictos entre el cuerpo docente de la Facultad de Ciencias Médicas y los sectores aglutinados en el CMA, quienes presionaron para que el Hospital de Clínicas tuviera una conducción conjunta entre la Municipalidad de la ciudad y aquella facultad ${ }^{10}$.

También el conflicto que rodea a la ley 6026 posee como protagonistas a médicos que provienen de los grupos antagónicos mencionados con anterioridad. Eliseo Cantón se asumió a sí mismo como un digno miembro del cuerpo docente de la Facultad de Ciencias Médicas y sostuvo que toda su labor apunto únicamente al engrandecimiento de la escuela médica local. En 1908, Cantón invocó en el parlamento la adhesión del cuerpo docente de esa facultad a su propuesta. En 1915, afirmó que las críticas de Dickman ofendían su autoridad como miembro del cuerpo docente. Por su parte, Enrique Dickman, activo miembro del Centro de Estudiantes de la Facultad de Medicina y del CMA, cuestionó la autoridad de Cantón como representante del «charlatanismo científico». Sin embargo, hay una diferencia clave con la configuración política que presentaba este escenario veinte años atrás: si a inicios de la dé-

gobernar. La construcción histórica de la profesión médica en Buenos Aires, 1852-1886, Madrid, CSIC, pp. 1-57. También puede verse SouZA (2005), pp. 44-82.

8 Penna, J. y Madero, H. (1910), La Administración Sanitaria y Asistencia Pública de la ciudad de Bs. As, Buenos Aires, p. 129; SouZA (2005), p. 149.

9 Ibíd., p. 67.

10 Esta situación conflictiva queda ilustrada en la fundación de un centro de atención de pequeñas dimensiones que se conoció con el nombre de policlínico del CMA, en el mismo año en que el cuerpo docente de la Facultad de Medicina pide al gobierno de la provincia de Buenos Aires la creación de un nuevo Hospital Escuela, esto es, en 1878. Las autoridades provinciales entregaron el edificio en julio de 1880. En los hechos, en el acto de su inauguración fue presentado como centro de atención de los heridos producidos en los tres combates en que se dirime el conflicto entre las milicias provinciales y las tropas nacionales. Ibíd., p. 75. 
cada de 1880 los grupos descontentos con el cuerpo docente de la Facultad de Ciencias Médicas no poseen la influencia política suficiente para opacar sus propuestas en la Cámara de Diputados, a inicios de la década de 1910 tal situación ha variado sensiblemente. Los sectores descontentos con la conducción de la Facultad de Ciencias Médicas hacen uso de esta capacidad para demostrar su posición de disidencia.

\section{ClíniCA EN El PARlamento: MÉdicos CONSERVAdORES VERSUS MÉdicos SOCIALISTAS}

En este punto se hace necesario concentrarnos en los protagonistas de la contienda científica y política por la sanción y posterior pedido de derogación de la ley 6026. Ciertamente, Eliseo Cantón y Enrique Dickman no son los únicos actores relevantes implicados en el ciclo de esta ley. Una cantidad importante de diputados —incluso, varios «diputados médicos»— acompañaron las presentaciones de ambos contendientes. El protagonismo de ambos médicos a lo largo del debate no debe omitir la existencia de una compleja trama de relaciones entre actores individuales y colectivos en la que se inscriben las biografías profesionales y políticas de los mismos. Así, se verá que actúan de forma convergente motivos tanto personales como colectivos, ambos motivados por la adscripción a grupos enfrentados política e ideológicamente dentro de la profesión y por la pertenencia a fuerzas políticas enfrentadas en la escena política nacional.

La focalización sobre Cantón y Dickman se ha realizado a partir de criterios empíricos y metodológicos que conviene presentar. En términos empíricos, la importancia de estos personajes se puede apreciar a través de la lectura secuencial de las páginas que abarca el ciclo de la ley 6026 en los Diarios de Sesiones de la Cámara de Diputados entre 1906 y 1917. Ahora bien, no sólo protagonizaron el debate en el parlamento, como diputados nacionales, sino que también expresaron conflictos médicos e ideológicos que trascendieron dicha escena institucional para alcanzar la esfera pública. En términos metodológicos, se ha privilegiado el momento de confrontación explícita entre ambos médicos, que se produce entre 1915 y 1917, dado que este recorte hace posible apreciar con nitidez los puntos de coincidencia y desencuentro entre las distintas miradas o cosmovisiones médicas ${ }^{11}$.

11 El presente artículo no intenta establecer qué cosmovisión es más verdadera; por el contrario, busca identificar las confrontaciones y los consensos entre los protagonistas del 
¿Quién fue Eliseo Cantón? Fue el autor e impulsor del proyecto de ley en la Cámara de Diputados, entre 1906 y 1910. Luego de la acusación realizada por Enrique Dickman en 1915, fue su obsesivo defensor. Cantón inició sus estudios en la Facultad de Medicina de Córdoba y terminó sus estudios en la Facultad de Ciencias Médicas de la UBA en 1886. En ella ocupó distintos cargos docentes, entre los que cabe señalar la titularidad de la materia Clínica Obstétrica; fue miembro de la Academia de Medicina y, entre 1906 y 1912, decano de la Facultad de Ciencias Médicas. Es decir, Cantón fue un digno representante de la «corporación docente». En términos políticos, desde fines de la década de 1880 se vinculó al roquismo y ocupó distintos puestos legislativos en representación del Partido Autonomista Nacional, también conocido como «partido conservador»: fue senador nacional por la provincia de Tucumán en dos períodos consecutivos, y luego fue diputado por la Capital Federal desde 1904 hasta $1911^{12}$. Fue vicepresidente de la Cámara de Diputados durante 1907 y presidente de la misma en 1908. Fue en este momento cuando logró sustanciar su proyecto de construcción de un hospital central de clínica, episodio que más tarde Dickman señalará como parte de sus manejos autoritarios y fraudulentos. Escritor prolífico de una importante cantidad de libros, artículos y observaciones clínicas, pueden destacarse su Historia de la Medicina en el Río de la Plata y La historia de la Facultad de Medicina de la Universidad de Buenos Aires ${ }^{13}$. En ambas obras, compuestas de varios volúmenes, se propone historiar el desarrollo de la medicina desde la llegada de los españoles hasta $s u$ presente, describiendo los años en que la escuela médica de Buenos Aires estuvo bajo su conducción como los de mayor esplendor. En 1916 escribió una serie de textos tendientes a defender el proyecto de ley presentado y, al mismo tiempo, a «desenmascarar» la acusación del «triunvirato médico socialista». En ellos, Cantón reconoce con orgullo que, durante los conflictos de 1904, impulsó la moción (triunfante por unanimidad) para que la facultad, por vía del poder ejecutivo, expulsara a los catedráticos socialistas Juan B. Justo y Nicolás Repetto. También reconoce que en ese mismo

debate y los grupos que ellos representan, tanto en cuestiones conceptuales como en las estrategias argumentativas desplegadas. Sobre esta perspectiva, ver SHAPIN, S. y SCHAFFER, S. (2005), Leviathan and the Air-Pump, Princeton, Princeton University Press, pp. 6-7.

12 Cfr. Pérgola, F. y Sanguinetti, F. (2000), p. 65. Tanto desde el punto de vista político como de su cosmovisión médica, la filiación conservadora de Cantón se puso en evidencia en su concepción gradualista de las transformaciones sociales y en su defensa del principio de autoridad sobre el que se configuraba el orden dominante, tanto en la arena política nacional como en la escuela médica en la que ejerce su profesión.

13 Ibíd., pp. 143-144. 
período estuvo detrás de la denegación del primer premio al estudiante del año al joven estudiante socialista Enrique Dickman, aduciendo que esta mención era para médicos argentinos.

El propio Cantón habilita una lectura del debate en torno a la ley 6026 como parte de los conflictos existentes entre el claustro de alumnos y las autoridades de la Facultad de Ciencias Médicas. En efecto, bajo el esclarecedor epígrafe de «Dickman sin medalla», el autor afirma en un texto de 1918 que:

Alguien observará, con razón, que en estos documentos no aparece para nada el nombre del Dr. Dickmann como para explicar la razón de ser de su actitud airada contra el policlínico y su autor. Pero aseguramos que existen dos, más que suficiente para explicarla, y descubrir a sus instigadores: 1) Sus vínculos de solidaridad sectario y gratitud personal para el Dr. Justo, quien cuando era profesor de la Facultad, pidió y obtuvo del entonces decano Dr. Uballes, el nombramiento del alumno Dickmann para ayudante del laboratorio de histología a fin de que pudiera terminar su carrera médica. ¿Habría algo de más natural que el actual señor diputado Dickmann, 'que de la nada ha llegado a serlo todo' (parodiando la frase de su exordio), se oponga al policlínico que pide la Escuela en que se instruyó, cuando ya no necesita de ella, si así venga y sirve a su protector? 2) Por no habérsele acordado la medalla de oro con que la Facultad premia al mejor alumno, de cada curso.

Luego de presentar una transcripción del acta de sesión del Consejo Directivo del 20 de octubre de 1910, donde se otorga el premio «Medalla de Oro» a Manuel Rosso (con nota promedio 9.37) y el «Diploma de Honor» a Dickman (con nota promedio 9.58), Cantón explica que:

La razón que como profesor tuve, y que posiblemente tuvieron la mayoría de los catedráticos que votaron a favor del Dr. Manuel Rosso, fue la de que, la «Medalla de Oro» debe acordarse al alumno argentino que una, a las mejores calificaciones, la consagración y la disciplina moral que augure la corrección de procederes, como hombre y como médico, en el seno de la sociedad en que debe actuar. Los hechos posteriores, incluso los de este debate, han demostrado con cuanto acierto procedió el cuerpo de profesores al acordar la medalla de oro, correspondiente al curso de 1904, a la persona que lo hizo ${ }^{14}$.

Por su parte, ¿quién fue el diputado que cuestionó en forma explícita en la Cámara de Diputados de la Nación a una figura tan poderosa y respetada de-

14 Cfr. CANTón, E. (1918), ¡Abajo la calumnia! El policlínico José de San Martín y el informe de la comisión parlamentaria. Falsedad de los cargos, Buenos Aires, Talleres Gráficos de L.J. Rosso y Cía., pp. 170-172. 
ntro de la clerecía médica local como lo era Eliseo Cantón? Entre fines de la década de 1890 e inicios de la siguiente, Enrique Dickman fue estudiante de la Facultad de Ciencias Médicas de la UBA. Durante este período, fue un activo participante en la creación del Centro de Estudiantes de dicha facultad en 1900 y representó al grupo de estudiantes y graduados opositores al cuerpo docente que conducía su vida institucional, al que pertenecía Eliseo Cantón. Como ya se ha expuesto, fue premiado con el diploma de honor, vale decir, con el segundo puesto al estudiante destacado de 1904, luego de haber obtenido las calificaciones más altas de su grupo y, por esto, merecer el primer premio que era la medalla de oro. Fue redactor de la revista médica y gremial del Centro de Estudiantes, editada desde 1900 y fusionada, desde 1913, con los Anales del Círculo Médico Argentino. Fue miembro del centro socialista Ciencia y Trabajo desde mayo de $1897^{15}$. Con posterioridad al primer lustro del siglo, fue miembro y director del periódico socialista La Vanguardia. El joven médico socialista escribe en este diario las primeras notas sobre la corrupción que rodea la elección del proyecto de hospital central en 1910, en momentos en que una comisión nombrada por el gobierno y dirigida por el propio Eliseo Cantón se proponía elegir uno de los ocho proyectos finalistas para dar forma al «Policlínico José de San Martín». Dickman ingresó al cargo de diputado en las elecciones de fines de 1913 como representante del Partido Socialista, luego de una primera presentación fallida en 1910. En la Cámara de Diputados, junto con Nicolás Repetto y Juan B. Justo, Enrique Dickman fue parte del grupo que los diputados conservadores $-\mathrm{y}$ en especial los diputados médicos como Eliseo Cantón- denominaron el «triunvirato médico socialista» ${ }^{16}$.

\section{El CiClo institucional DE LA LEY 6026}

El ciclo de la ley 6026 en la Cámara de Diputados de la Nación se inicia en 1906 y culmina en 1917. Entre estas fechas emerge en reiteradas ocasiones el tratamiento del tema «Policlínico José de San Martín». Estas apariciones dejan entrever distintas sensibilidades respecto del tema del policlínico y, más en general, de la problemática hospitalaria en la ciudad hacia inicios del siglo XX. Entre la primera fecha y el año 1908, el diputado Eliseo Cantón presentó tres proyectos distintos sobre el mismo policlínico, obteniendo la sanción para

15 IÑIgo Carrera, N. (1996), Documentos para la Historia del Partido Socialista, Tandil, IEHS, p. XVIII.

16 Cfr. CANTÓn (1918), p. 179. 
su construcción en el año 1908, cuando ocupaba el cargo de presidente de la Cámara de Diputados ${ }^{17}$. Entre junio y julio de ese año, en dos sesiones maratónicas, el último de sus proyectos se transformó en la ley 6026, que mandaba a construir uno de los «hospitales centrales» $\mathrm{u}$ 《hospitales escuelas» mas grandes de su tiempo ${ }^{18}$. En el apartado siguiente nos centraremos en las características tecnológicas y médicas del hospital soñado por Cantón.

Entre 1909 y fines de 1910 se aprecia una intensa actividad tendiente a sustanciar la construcción del nosocomio. Para el autor del proyecto, se debía al menos colocar la piedra fundamental de la obra como parte de la fastuosa conmemoración del centenario de la revolución de mayo de $1810^{19}$. Este objetivo no fue posible debido a la inminencia de esta celebración. A cambio, se concretaron algunos pasos fundamentales para la construcción. Se llevaron adelante las primeras expropiaciones de tierras sobre las que se asentaría el hospital y, al mismo tiempo, se comenzó a desviar un porcentaje de las recaudaciones de la lotería pública para financiar los gastos de la construcción ${ }^{20}$. Se llamó a concurso de proyectos para la construcción del hospital y, en forma paralela, se designó la comisión que debía evaluar los ocho proyectos finalistas sobre un total de catorce proyectos presentados. Hubo oferentes internacionales. Por ejemplo, uno de los proyectos finalistas llamado, «Estrella de oro con Perlas», provenía de la legación de Paris; otro que no arribó a la final provenía de Alemania y se titulaba «Gloria de San Martín»»

La ingente magnitud de dinero necesaria para financiar la construcción del hospital, estimada en más de 15000000 de pesos, justificó la creación de una comisión con representantes del poder ejecutivo y miembros de la Facultad de Ciencias Médicas para realizar la selección ${ }^{22}$. La elección del proyecto ganador a inicios de 1910 no fue unánime y se produjeron fuertes disidencias entre los representantes de la Facultad de Ciencias Médicas y el miembro que representaba al poder ejecutivo, el famoso arquitecto Norberto Buschiazzo. Mientras que los primeros siguen la opinión de Eliseo Cantón y eligen el proyecto llamado «Aire y Luz», el segundo presenta un escrito a los miembros

17 Diario de Sesiones de la Cámara de Diputados de la Nación Argentina (1908), p. 145.

18 Ibíd., p. 577.

19 Ibíd., p. 210.

20 Diario de Sesiones de la Cámara de Diputados de la Nación Argentina (1915), pp. 107-109.

21 Cfr. CANTÓN (1918), pp. 85-86.

22 Diario de Sesiones de la Cámara de Diputados de la Nación Argentina (1915), pp. 110-111. 
del flamante gabinete nacional (entrante hacia fines de 1910) en donde detalla una importante cantidad de errores existentes en el proyecto ganador, entre ellos, el de prestar mayor atención a los aspectos artísticos del policlínico que a su disposición clínica. También afirma haber sido marginado de la decisión y no haber votado por el proyecto de policlínico ganador ${ }^{23}$.

Es en ese mismo momento que se aprecia un fuerte viraje en la buena predisposición que había hacia el policlínico entre los propios partidarios de Cantón. Roque Saenz Peña — nuevo presidente de la Republica- estudió personalmente el informe de Buschiazzo y ordenó «enterrar el asunto» del policlínico, bajo la sospecha de corrupción y el peligro de que se transformara en un escándalo político ${ }^{24}$. También en la prensa porteña se publicaron comentarios críticos. En algunos casos se ironizaba sobre las «propiedades elásticas» del proyecto, dado que era mayor que la superficie asignada para su construcción. El diario El País se hizo eco de las ironías más mordaces contra el proyecto del Dr. Cantón:

El asunto del policlínico continúa agitando la opinión. Hasta ha encontrado quien se adhiera al fallo del jurado: mejor así, sin embargo, porque el debate, la polémica, que significa luz, son preferibles a la oscuridad, con cuya complicidad se han hecho y siguen haciéndose tantas cosas vedadas. Los entendidos ya han desahuciado el proyecto premiado 'Aire y Luz': el análisis severo, científico, ha señalado sus defectos enormes - y en esto merece un aplauso de colegas de 'la patria degli italiani' —, y el humorismo porteño, caricaturizando esos defectos, ha sabido graciosamente poner la cosa en solfa olvidando por un instante que el asunto es grave, como que entraña la pérdida de varias decenas de millones para el contribuyente. Ese solo hecho de haber sido proyectado el plan del policlínico sobre un área superior en ocho mil a la asignada para su construcción, es curiosísimo: pero mucho más graciosa es la explicación que el doctor Cantón le ha descubierto al error, al sostener que llevando el policlínico del plano a la realidad de la construcción, 'se achicara'. Como 'truvaille' este policlínico elástico es impagable. Se podría enseñarlo en las ferias al lado del hombre cañón o la mujer foca ${ }^{25}$.

Cinco años después - hacia mediados de 1915 - reaparece el tema del policlínico en la Cámara de Diputados, esta vez rubricado por el escándalo. Enrique Dickman realiza un pedido de interpelación al Ministerio de Hacienda

23 El informe de Buschiazzo citado por Dickman es una crítica contundente, tanto al proyecto de policlínico de Cantón como al funcionamiento de la comisión que lo eligió. Cfr. Diario de Sesiones de la Cámara de Diputados de la Nación Argentina (1915), pp. 124-125.

24 Ibíd., p. 126.

25 Cfr. El País, 21 de febrero de 1910, p. 7. 
para que se de cuenta ante la cámara del estado de avance del policlínico ${ }^{26}$. Dicho pedido es respondido personalmente por el ministro Enrique Castillo, quien afirma que el proyecto quedó en la fase de expropiación de tierras debido al desvío de los fondos necesarios para su construcción a otras necesidades presupuestarias en 1912. Castillo señaló que las modificaciones de la economía por el estallido de la guerra en Europa recomendaban la suspensión momentánea del proyecto o su realización bajo otras condiciones. Aprovechando esta afirmación, el diputado socialista hizo el pedido de derogación de la ley 6026, dando lugar a una acalorada exposición en la que se denunció al ex diputado Cantón - y por ese entonces decano de la Facultad de Ciencias Médicas de la UBA - por malversación de fondos y, en general, a todo el proyecto como fraudulento, producto del orgullo «megalómano» de Cantón, un verdadero «colazo del viejo régimen, que se ha caracterizado no solo por escrutinios falsos, sino por grandes escándalos financieros $\gg^{27}$.

La contundencia de la acusación y la minuciosidad de la investigación llevada a cabo por Dickman, abrió una sucesión de excusaciones por parte de aquellos diputados que habían participado en las distintas comisiones que evaluaron el proyecto o, también, que habían avalado con su voto la transformación de proyecto a ley en 1908. No faltaron las ofensas e, incluso, las invitaciones a resolver la acusación en el «terreno de la caballerosidad» promovidas por aquellos diputados unidos a Eliseo Cantón por un vínculo de camaradería ${ }^{28}$. Otros miembros de las filas conservadoras - no tan cercanos al ex diputado y menos apasionados - buscaron atenuar la magnitud del escándalo a través de la formación de una comisión encargada de estudiar la veracidad de la denuncia.

Esta comisión se efectivizó entre 1915 y 1917 y dio un veredicto absolutorio a la figura de Eliseo Cantón ${ }^{29}$. Su funcionamiento fue denunciado por par-

26 Diario de Sesiones de la Cámara de Diputados de la Nación Argentina (1915), p. 25.

27 Ibíd., p. 111.

28 En la sesión del 28 de mayo de 1915, es decir, en la sesión siguiente a la que Dickman pidiera la derogación de la ley 6026, el diputado Arce, al comentar las afirmaciones realizadas por el diputado socialista, juega con la idea de que los miembros del grupo que apoyan a Dickman están entre los diputados que no aceptan la «vía caballeresca» como camino para dirimir las ofensas personales realizadas en la cámara. Cfr. Ibid., p. 147.

29 La comisión parlamentaria destinada a investigar las afirmaciones del diputado Dickman estuvo activa entre 1915 y 1917, momento de la emisión del informe cuestionado por los diputados de la bancada socialista. Su importancia queda reflejada en que es la única comisión ad hoc enunciada formalmente en el indice de los diarios de sesiones entre ambos años. A pesar de las pocas veces que esta comisión se reunió, produjo una cantidad importante de documentación, que según el diputado socialista llegaba a las 4500 hojas (algo más de 80 
cialidad en el manejo de la información, y por elaborar un veredicto sin la participación del representante socialista en la misma. En efecto, el diputado Mario Bravo afirma que se le denegó la documentación para el estudio de la causa a través de excusas poco verosímiles ${ }^{30}$. También afirmó que entre 1916 y 1917 esta comisión permitió a Cantón extraer información confidencial de la causa para poder publicar una serie de textos en donde defiende en tono polémico y agresivo el proyecto de ley. El más exacerbado, titulado «iAbajo la Calumnia! El Policlínico José de San Martín y la Difamación del Socialismo Ruso», fue publicado en 1916 como un texto suelto de unas pocas carillas, y transformado en 1917 en un libro de casi 250 páginas. En estos escritos, Eliseo Cantón afirmó que el motivo de la denuncia es la venganza por la expulsión de los médicos socialistas de la Facultad de Ciencias Médicas en 1904, a quienes apodaba ahora de «socialistas internacionalistas», y acusaba de no respetar las autoridades académicas de la facultad. Al mismo tiempo, volvía a defender la necesidad de llevar adelante el proyecto ley.

\section{EN BUSCA DE UN HOSPITAL CLÍNICO CENTRAL PARA BUENOS AiRES}

Cantón y Dickman confrontaron explícitamente sobre el tipo de hospitales y la cantidad de camas por habitante para la ciudad de Buenos Aires. Era un momento de acelerado crecimiento demográfico. Ninguno de los dos dudó de la importancia del hospital para el aprendizaje del «apostolado de la medicina». Sí se pueden apreciar, en cambio, nociones diferentes sobre el compromiso que debería unir al médico clínico con el contexto social en que se inscribe su práctica. Cantón apeló en reiteradas ocasiones a una noción abstracta de la población porteña, que en su particular mirada recibiría con sumisión los beneficios de la tecnología hospitalaria, puestos fuera de duda por el mero hecho de encarnarse en un modelo de nosocomio exitoso para las escuelas médicas europeas. Dickman dejó entrever una preocupación por comprender la idiosincrasia de la población y de sus representaciones sobre la enfermedad y el hospital y, en tal sentido, se orientó a un sistema hospitalario que no resultara invasivo, que no atemorizase a los sectores populares.

Presentada esta tensión que atraviesa el debate, nos dedicaremos en lo que sigue a algunos puntos de confrontación existentes entre ambos proyectos de

\footnotetext{
expedientes). Dicha comisión estuvo integrada por varios miembros como consecuencia de la renovación de los cargos en la cámara producida en el año 1916. Ibid. (1917), p. 104.

30 Ibíd., pp. 100-101.
} 
medicalización. Para comprender de forma contextualizada el contraste entre las miradas polares del médico socialista y del ex decano de la facultad, veamos qué tipo de referencias a la problemática hospitalaria pueden encontrarse en la prensa de las últimas décadas del siglo diecinueve. Por una parte, en los años previos a la creación del Hospital de Clínicas de Buenos Aires en 1883, son frecuentes las menciones a la necesidad de modificar los hospitales de antigua data e, incluso, de cambiar el antiguo Hospital General de Hombres por un hospital clínico moderno. Luego de 1883 se suman aquellas discusiones que aluden a distintos problemas del orden interno del hospital de clínica, como, por ejemplo, la ausencia de algunas clínicas especializadas o la necesidad de un número mayor de camas para las salas disponibles. También existen varias referencias a la ausencia de instrumental específico, como estetoscopios, sondas, pinzas, piezas para el museo del hospital, microscopios para las salas que comenzaban a funcionar como incipientes laboratorios, etc. Sin embargo, no se debate sobre modelos alternativos de hospitales para la ciudad, ni se formulan críticas explícitas a cuestiones de tecnología hospitalaria.

Por otra parte, las preguntas formuladas al material empírico no han recortado en forma caprichosa un tema especial, como podría haber sido, por ejemplo, el hospital y su modelo arquitectónico, sino que han respetado el encadenamiento de significados que los propios diputados dieron en sus argumentos a los temas relevantes de la vida hospitalaria. En otras palabras, en ambas exposiciones se habla de un número importante de temas relacionados con el hospital, como la cantidad de camas por población, la cantidad de camas en una sala clínica, la cantidad de salas dentro de un hospital, la cantidad de metros cuadrados que deben poseer los policlínicos modernos, el funcionamiento de las dependencias subsidiarias del hospital, etc.

¿Qué modelo de nosocomio contempla Eliseo Cantón en sus propuestas? ¿Qué problemas vendría a solucionar la creación de dicho nosocomio? El modelo de hospital anhelado por Cantón es lo que él denomina en reiteradas ocasiones como «hospital central», al que suele referirse familiarmente como «laboratorio de personas» ${ }^{31}$. El concepto de hospital central remite a un edificio de dimensiones imponentes para la época, que ocuparía un espacio de cuatro manzanas centrales en la ciudad y por lo tanto, de alto valor económi$\mathrm{co}^{32}$. El hospital tendría un subsuelo y dos pisos de altura; en este espacio, se

31 Ibíd. (1908), p. 548.

32 La ubicación del policlínico mencionada en el artículo 2 ocupaba cuatro manzanas, que aún en la actualidad son centrales en el trazado urbano de la ciudad de Buenos Aires. Las calles mencionadas en el proyecto de ley presentado por Cantón conservan su mismo nombre, 
debería distribuir un «sistema» de veinte institutos orientados al estudio y atención de clínicas especificas. Este sistema de institutos se identifica con el programa de estudios médico-clínicos vigente luego de 1883 en la escuela médica local, al que se vendrían a sumar dos institutos dedicados a la clínica obstétrica, ya que dichos estudios carecieron durante un tiempo importante de salas donde desarrollarse hasta la creación de la clínica obstétrica en 1901. Finalmente, el número de camas por instituto sería de $60 \mathrm{y}$ el total para todo el hospital de 1200, dato que significaría un crecimiento importante respecto de las 350 camas existentes en el Hospital de Clínicas de 1883. Según afirmó Cantón en reiteradas ocasiones, las camas tendrían cerca de 81 metros cuadrados de espacio circundante, que era una medida aproximada a los estándares fijados para la construcción de policlínicos. El hospital en su totalidad tendría aproximadamente 97000 metros cuadrados, cifra que se prestó a polémicas mientras se debatieron los proyectos y, una vez sustanciada la ley, fue criticada con dureza por la prensa porteña. En el texto del proyecto de ley se lee:

Articulo 1, El poder ejecutivo hará construir para la Facultad de ciencias médicas un policlínico que se denominará «José de San Martín». Artículo 2, El establecimiento será construido en las cuatro manzanas y calles intermedias que limitan las calles: Córdoba y Charcas, Junín y Azcuenaga, las que al efecto se declaran de utilidad pública. Articulo 3, El poder ejecutivo hará efectiva la expropiación de tres de aquellas manzanas de tierra y licitará la obra de conformidad a las siguientes bases: a) Los proponentes presentarán los planos, presupuestos y especificaciones dentro del plazo de seis meses al Poder Ejecutivo para su aprobación o rechazo, b) Los planos y presupuestos serán pasados a informe de una comisión compuesta de tres consejeros de la facultad de ciencias médicas, y de dos arquitectos de reconocida competencia, la misma que tendrá a su cargo la dirección e inspección de todas las obras, c) El plan general del policlínico responderá al sistema de institutos separados, cuyo número será de veinte: cuatro (4) para clínica médica; tres (3) para clínica quirúrgica; dos (2) para clínica obstétrica; uno (1) para patología externa; uno (1) para patología interna; uno (1) para clínica pediátrica; uno (1) para semiología; uno (1) para clínica dermatológica y sifilográfica; uno (1) para clínica ginecológica; uno (1) para clínica oftalmológica; uno (1) para clínica neurológica; uno (1) para clínica otorino-laringológica; uno (1) para clínica genito-urinaria; uno (1) para hidro electro terapia y laboratorio central, d) El promedio de capacidad de los institutos para clínica será de sesenta camas, e) Las construcciones se levantarán

\footnotetext{
a excepción de la segunda que ha pasado a llamarse Marcelo T. de Alvear. Estas calles delimitan las cuatro manzanas en las que se encuentran hoy la Plaza Houssay, la Facultad de Medicina, la Facultad de Farmacia y Bioquímica y la Facultad de Ciencias Sociales de la UBA, y el actual Hospital de Clínica.
} 
tres metros adentro de la línea de las calles, y no tendrán más de dos pisos y un subsuelo, [...] Artículo 7, Comuníquese al Poder Ejecutivo. Eliseo Cantón ${ }^{33}$.

El policlínico «José de San Martín» fue comparado a un número importante de policlínicos centrales europeos. El autor del proyecto dio por sentado, como un «hecho de peso científico», que los policlínicos centrales han sido pensados y construidos como una verdadera superación de los hospitales existentes hasta mediados del siglo XIX. Cantón justificó el importante monto de dinero a invertir en el policlínico señalando que las naciones europeas que marchan a la «vanguardia de la ciencia» en materia médica han logrado sostener esa condición mediante grandes inversiones de dinero en hospitales clínicos centrales. Este modelo de policlínico traía consecuencias de importancia para los valores médicos de la época; la primera de ellas, es que había permitido articular - en los países mencionados por Cantón en su exposición - un sistema de hospitales urbanos, suburbanos, y extra urbanos, con los cuales cubrir las demandas de salud de un espectro importante de territorios, personas y patologías. La segunda consecuencia importante es que aumentaba el número total de camas para internación disponibles por cada país ${ }^{34}$.

Eliseo Cantón tomó como dato de importancia la correlación entre cantidad de camas por mil de habitantes que disponían en las principales ciudades europeas. Existía, según él, una verdadera relación de fuerzas en materia de camas hospitalarias a escala internacional a la que Buenos Aires debería sumarse. Esta ciudad poseía, según su propio cálculo, cerca de 2.2 camas cada mil habitantes para una población cercana a 1.150.000, mientras que Londres poseía 5.68 y Dublín 6.39, París 9.83, Berlín 3.98, Amsterdam 4.66, Madrid 4.00, entre otras. ${ }^{35}$ A su juicio, Buenos Aires carecía de un verdadero hospital central y la construcción del policlínico «José de San Martín» vendría a solucionar ambos problemas:

33 Diario de Sesiones de la Cámara de Diputados de la Nación Argentina (1908), p. 145.

34 Ibíd., p. 536.

35 El listado completo de capitales y camas mencionadas por Cantón fue el siguiente: «En Inglaterra hay por cada 1000 habitantes 5.68 camas; en Dublín, 5.39; en París 9.83; en Berlín 3.75; en Amsterdam (Holanda), 11.66; en Roma 18.28; en Nápoles, 12.62; en Milán, 17.28, en Moscú, 5.16; en San Petersburgo, 9.08; en Madrid, 4; en Estocolmo, 6.94; en Viena, 4.84; en Budapest, 8.26; en Nueva York, 3.30; ;en Buenos Aires, hay apenas dos por mil! Y, como bien decía el miembro informante, ¿será esto porque Buenos Aires es excesivamente sano y no necesita mayor número de camas? No, señor presidente. Quien conoce los hospitales, sabe perfectamente bien en qué forma se encuentran hacinados los enfermos: además de las dos clásicas hileras de camas, puestas a uno y otro lado de los salones, existe una tercera, desvalida en verdad porque está tirada en el suelo». Ibíd., p. 542. 
Yo sostengo que Buenos Aires no tiene, y necesita imperiosamente un hospital urbano, un hospital central. El único que hace las veces de tal es el muy pequeño Hospital de Clínicas con trescientas camas; y sacarlo de allí para llevarlo a la Chacarita, aunque se le agrande, es dejar desvestida a Buenos Aires de su traje más primordial, y es la única ciudad del mundo que nos ofrecería el extraordinario fenómeno de no tener ningún hospital central ${ }^{36}$.

En cuanto a la legitimidad de los argumentos, Cantón invocó en forma aleatoria la doble autoridad de su experiencia personal — diputado y catedrático-, además de los tratados internacionales especializados en materia de construcción hospitalaria. Entre estos últimos se destaca el libro La construcción de hospitales, de Vandelvelde, Lepage y Chaval, tres médicos de Bruselas. Este libro posee todos los atributos de un clásico de la materia para su época, a juzgar por el número de sus reediciones ${ }^{37}$. El sólido eje narrativo de Cantón extraía una notable cantidad de datos empíricos de esta bibliografía. Por su parte, los diputados que escucharon a Cantón en 1908 reconocieron la importancia del policlínico, incluso aquellos que realizaron alguna temprana crítica al proyecto. El círculo más estrecho de diputados al que pertenecía el entonces decano de la Facultad de Medicina señaló la deuda de gratitud que la ciudad poseía con su mentor. Desde este sector, Cantón fue igualado a los grandes médicos constructores de hospitales de Europa —en especial, al médico italiano Beccheli, fundador del policlínico Humberto I en Roma-y, al mismo tiempo, fue comparado con una figura antaño poderosa de la medicina local, como fue el ministro de Instrucción Pública y Culto Eduardo Wilde durante el primer gobierno de Julio A. Roca en los ochenta. Cantón no oculta su aspiración a un reconocimiento simbólico similar y, para ello, nada mejor que un hospital de dimensiones gigantescas como el policlínico San Martín, que eclipsara a Wilde y al ya «vetusto y achacoso» Hospital de Clínicas que aquel había conseguido. En cuanto a su retórica, como profesional que seguía los pasos de estas eminencias científicas, Cantón afirmó estar interesado solamente en el progreso real de la ciencia médica local, en la ardua tarea que la llevaría a equipararse con las potencias médicas internacionales. Así lo confesaba en respuesta a una moción de aplazamiento del proyecto formulada por un miembro de la comisión de hacienda de la Cámara de Diputados en 1908:

36 Ibíd., p. 548.

37 El diputado Cantón cita este libro al menos dos veces en sus intervenciones del año 1908. Lo trae a colación frente a los miembros de la Comisión de Hacienda y Obras Públicas $\mathrm{y}$, con posterioridad, a la hora de justificar la ubicación central del hospital en el trazado urbano de la ciudad. Ibíd. 
¿Qué importa que se aplauda mi proyecto y que se elogie injustamente a su autor, si con la moción de aplazamiento formulada se pretende pegarle un golpe de gracia en el momento en que va a surgir? ¿Qué importa todo esto? ¿Qué importa, aún más, que se me califique de entusiasta y hasta de fanático si todo se olvida, incluso que ha habido en todas las épocas de la historia fanatismos que han sido excusables? Y cuando estos fanatismos se han fundado en razones científicas y en nobles ideales, no sólo han sido excusables, si no justificables y hasta dignos de aplauso. En tiempos de las cruzadas, cuando dominaba el mundo del fanatismo religioso, los caballeros esforzados se lanzaban a la lid bajo la evocación de «por mi Dios, por mi rey, y por mi dama». En tiempos actuales, en que domina las naciones civilizadas el fanatismo por el saber, los hombres se incorporan a los debates parlamentarios reclamando para su fuero interno «por las ciencia, por la humanidad y por mis ideales»; por la ciencia, que persigue la verdad; por la humanidad, que aprovecha de sus conquistas; y por los ideales que dignifican la existencia y la especie humana ${ }^{38}$.

Como se podrá apreciar a continuación, tales intenciones no eran unánimemente aceptadas entre la comunidad de médicos locales, en especial, entre los médicos más jóvenes pertenecientes a instituciones médicas distintas a la academia y al cuerpo docente de la Facultad de Medicina.

\section{LAS CRÍTICAS DE ENRIQUE DiCKMAN A LA LEY 6026}

Dickman criticó el concepto de hospital central. Este modelo de «hospital monstruo» era poco eficaz por aquel motivo que Eliseo Cantón precisamente destacaba, esto es, el importante grado de centralización de sus actividades. Pero además era poco eficaz por los altos costos de mantenimiento y por su semejanza a otras instituciones de encierro, como las cárceles. Poco eficaz en sí mismo, también era poco eficaz en una ciudad como Buenos Aires hacia 1915, en la que sus sectores populares no asistían masivamente a los nosocomios, a pesar del sensible aumento de consultas e internaciones hospitalarias registradas desde fines de la década de 1880. Según Dickman, todo el proyecto era un «colazo del antiguo régimen que se acaba», situación que no titubeó en homologar a la vida cotidiana de la Facultad de Medicina. Tal comparación no era forzada, si se piensa que Cantón era al mismo tiempo diputado por el Partido Autonomista Nacional y decano de la Facultad de Medicina. Dickman afirmó que se habían malversado fondos públicos a través del manejo de precios fraudulentos en las tierras a expropiar y, en general, trató al decano de la facultad

38 Ibíd., p. 577. 
de charlatán, autoritario y habló de «rastacuerismo científico» al afirmar que las dimensiones del futuro policlínico no tendrían igual en el mundo.

En cuanto a las críticas referidas al modelo de nosocomio, Dickman complejizó la relación entre el hospital y el tejido urbano, dando lugar a una lectura algo distinta de las necesidades hospitalarias de la ciudad de Buenos Aires. En principio, cuestionó la idea de «hospital monstruo» como cumbre del saber médico. Se trataba de un concepto antiguo, afirmaba, que había fracasado en los países «centrales», donde tienen «tendencia a lo descomunal»:

Por otra parte, debo decir a los señores diputados que los hospitales grandes han fracasado aún en el país mismo donde han sido concebidos, en Alemania, donde han tenido la tendencia a lo colosal. Allí se han hecho hospitales colosales, como el Virchow, de dos mil camas, como el Eppendorf, de Hamburgo, de 2150 camas; pero hoy los autores alemanes están contestes, y así se puede leer en este libro (muestra uno) especie de enciclopedia de muy reciente publicación sobre materia hospitalaria, escrita por distinguidos profesores en la materia, que el hospital grande ha fracasado en la práctica. El hospital grande es más caro en su construcción; es más costoso de sostener, se administra peor, el enfermo está mal atendido. Al hospital grande el público le tiene mucha aversión, porque lo considera como una especie de cárcel o de cuartel en donde se entra para no salir más. En el hospital grande el público se pierde en sus visitas. En una palabra, el hospital grande es impopular, antihigiénico, antieconómico y anticientífico ${ }^{39}$.

El diputado socialista esta cercano a un servicio de salud diversificado en hospitales pequeños y focalizados. $Y$ en base a esta idea propone una relectura del dispositivo clínico porteño cristalizado en la década de 1880 con la ley 1284. Se deberían descongestionar los hospitales de los pacientes convalecientes y alojarlos en un hospital especial construido en las afueras de la ciudad, en tierras fiscales más baratas que las tierras elegidas por Cantón. Como complemento de este hospital ubicado en las afueras de la ciudad, se podrían construir hospitales pequeños dentro del tejido urbano con un mayor espacio para cada cama: «Lo que hace falta ahora son hospitales pequeños, cuyas ventajas convienen en reconocer todos los hombres de ciencia: hospitales urbanos de 200 a 400 camas, y hospitales suburbanos de 500 a 700, como maximum, con una superficie mínima de 100 metros cuadrados por cama $\rangle^{40}$. Esta disposición permitiría afrontar un viejo problema de la medicina local, como es la mala fama que tiene la hospitalización:

\footnotetext{
39 Ibíd. (1915), p. 118.

$40 \quad$ Ibíd., p. 118.
} 
Puedo afirmar que la población de la capital federal no tiene tendencia a hospitalizarse; le tiene horror a los hospitales. Ese horror proviene de una mala tradición, porque antes en los hospitales se consideraba al enfermo como un caso lindo: cuanto mas grave, cuanto mas insalvable, cuanto mas inoperable era el caso, mas lindo parecía a la ciencia: considerábase a los enfermos como cosas, como carne de hospital. Hoy, felizmente, la juventud universitaria, siguiendo la evolución y el ritmo del progreso general, tiene otras ideas y otros conceptos sobre su misión científica y sobre el fin a que se destinan los hospitales; pero en general la población tiene aún aversión y horror a los hospitales, con cierta razón, con ciertos fundamentos, sobre todo teniendo en cuenta que en la capital federal, donde se han invertido millones en expropiaciones de avenidas, en los hospitales no hay remedios ni elementos de curación suficiente para los enfermos ${ }^{41}$.

Si bien el miedo de los sectores populares a los hospitales excede nuestro foco de atención, conviene aclarar que se trata de un tópico de permanente preocupación para la profesión médica finisecular. El reconocimiento de las malas condiciones edilicias reinantes en los hospitales de Buenos Aires y del escaso apego que poseían los sectores populares a «hospitalizarse» aparece de forma recurrente en los periódicos médicos locales desde la década de 1870.42 Dickman no ignoraba que las transformaciones acaecidas en materia hospitalaria en la ciudad durante las tres décadas precedentes no gozaban de un reconocimiento generalizado en los distintos grupos de la sociedad civil porteña.

Así, una vez criticado el concepto mismo de hospital central, Dickman pasó a discutir la correlación entre camas y habitantes para una ciudad como Buenos Aires y, por otra parte, la cantidad de espacio necesaria para cada cama dentro de un hospital de las dimensiones pretendidas para el policlínico San Martín. La discusión de este punto era clave, pues se trataba de uno de

41 Ibíd.

42 A modo de muestra, transcribimos unas líneas de un artículo publicado en la revista del CMA en el año 1879. Refiriéndose al pobre que arriba enfermo a un hospital, el joven médico Aníbal Torino expresa: "[...] estos enfermos cuyo organismo debilitado por los sufrimientos, por la mala alimentación y abatida su moral por tanta miseria, se hallan muy frecuentemente privados de esa suma de energía necesaria para reaccionar contra las nuevas causas deletéreas con que tropiezan en el hospital, lo que hacen en último extremo ¿Por qué esta aversión para entrar al Hospital? Es porque los hospitales actuales se parecen mucho mas a tumbas que a asilos de beneficencia; es porque en estos vastos monumentos adonde están reunidos los enfermos de toda especie, piso sobre piso, se respira aire tibio y pestilencial. Es porque el pueblo sabe que son malsanos, que muchos entran allí indispuestos y ya no salen vivos. He ahí por qué el pobre tiene horror al hospital, he ahí por qué no entra sino cuando la necesidad y la falta absoluta de recursos lo obligan". Cf. ToRINO, A. (1879), Anales del Círculo Médico Argentino, tomo II, p. 218. 
los motivos centrales pretextados por el autor del proyecto y, en tal sentido, el diputado socialista hizo una presentación contundente de los «errores de juicio» y de interpretación de Cantón.

Según Dickman, Buenos Aires no era una ciudad que careciera de camas $\mathrm{y}$, por ende, no estaba necesitando de un «hospital monstruo». El dato sobre el número de camas expuesto por Eliseo Cantón era fraudulento, ya que no contemplaba la totalidad de camas existentes en la ciudad. En otras palabras, para Dickman no había un consenso tan unánime - como el que reclamaba Cantón- respecto del número de camas por mil habitantes. El cálculo de las mismas sin duda era un dato de gran importancia, que dependía de un número de circunstancias y no de un simple juego aritmético. Entre otras variables a considerar, influían «los hábitos de la población para hospitalizarse», el «bienestar de la población», si la «la época era de bonanza o de crisis», si la «ciudad era industrial o no». Bajo estos parámetros, la correlación entre camas y habitantes variaba en forma sensible, había ciudades que necesitan 12 camas por cada mil habitantes, y había otras ciudades que les bastaba con 4 o 5 cama por mil habitantes. Frente a este panorama, no había que construir «hospitales monstruos», sino reordenar las capacidades hospitalarias disponibles en la ciudad de Buenos Aires:

Se ha dicho también en el seno de la honorable cámara que se necesitaba un gran hospital, y se lo necesitaba porque en Buenos Aires faltaban camas, porque las camas de los hospitales apenas alcanzaban a 2000, y no habiendo camas en los hospitales, era indispensable y urgente construir un gran nosocomio con propósitos de enseñanza y también con propósitos de hospitalización. Yo me he ocupado señores diputados, de conocer con exactitud el número de camas que hay en la capital federal, y veo con dolor que este dato dado en el seno de la honorable cámara también ha sido falso ${ }^{43}$.

De acuerdo con Dickman, es falso que en la Capital sólo haya 2000 camas. Según la asistencia pública hay 11900 camas: 4655 municipales, 6060 nacionales y 1275 en hospitales extranjeros. Si bien cree que estas cifras reflejan que faltan camas para los enfermos, las necesidades perentorias son otras:

Yo he visto que en los hospitales falta alcohol, tintura de yodo, gasa y algodón para las curaciones; yo he visto médicos jóvenes y laboriosos, como el hijo del señor diputado del Valle, sacar dinero de su bolsillo para comprar alcohol y tintura de yodo para curar en la sala. Y si no hay elementos para sostener los actuales hos-

43 Diario de Sesiones de la Cámara de Diputados de la Nación Argentina (1915), p. 117. 
pitales, ¿cuál es el problema urgente? ¿Construir nuevos hospitales o dar dinero para sostener los existentes? Pero hay más: yo creo que el número de camas es casi suficiente para la población de la capital federal; lo que hay que hacer es una cosa muy distinta a construir hospitales monstruos, que han fracasado en todas partes del mundo, como van a ver los señores diputados; lo que se necesita es retirar de los hospitales comunes la cantidad de tuberculosos que en ellos se asisten, porque constituye un peligro permanente para los enfermos que no lo son y para ellos mismos al estar hospitalizados en hospitales comunes ${ }^{44}$.

Para Dickman, la inversión en construcciones hospitalarias debía concentrarse en el hospital Tornú, por aquellos años el hospital municipal destinado especialmente a los enfermos de tuberculosis:

Hay un hospital municipal, el hospital Tornú, edificado en un terreno de 72.000 metros cuadrados, que tiene 360 por cama, destinado a tuberculosos; y lo que habría que hacer es construir allí otros pabellones para alojar a todos los tuberculosos, que se asisten en todos los hospitales, construir cerca de la Capital, algún gran establecimiento para convalecientes ${ }^{45}$.

Finalmente, en cuanto al tipo de atención y al lugar en que deberían edificarse nuevos edificios:

En los hospitales, los enfermos se asisten rápidamente por que se necesita la cama y los enfermos salen apenas repuestos de sus dolencias en un estado en que muchos de ellos no pueden ir a sus casas ni dedicarse al trabajo; lo necesario es hacer un gran edificio sobre un terreno vecino a la capital para descongestionar los hospitales comunes de los convalecientes y al cual puedan ir durante veinte días, un mes o un mes y medio a restablecerse en pleno sol y aires, a fin de que puedan volver a sus hogares aptos para el trabajo ${ }^{46}$.

Dickman afirmó que no solo era erróneo el número de camas señalado en la presentación de Cantón, sino también el cálculo realizado sobre la cantidad de metros cuadrados por cama. La cantidad de metros cuadrados que el proyecto de 1908 contemplaba para cada cama del policlínico era de 81 metros cuadrados, pero según Dickman era este un dato que contrariaba a las autoridades internacionales en materia de construcción hospitalaria. En efecto, la cantidad

\footnotetext{
44 Ibíd., pp. 117-118.

45 Ibíd., p. 118.

46 Ibíd., p. 119.
} 
de metros cuadrados por cama era sensiblemente mayor a la presentada por Cantón. Se necesitaban al menos 100 metros cuadrados por cama si se trataba de un «hospital chico», y más de cien si se trataba de un «hospital grande»:

Para la construcción de hospitales, señores diputados, hay leyes y reglas perfectamente establecidas, conocidas y estudiadas por autoridades científicas del mundo entero. Se sabe que no se puede construir un hospital moderno sin infringir las reglas más elementales de higiene sanitaria, sin tener una superficie de cien metros para cada cama de hospitalización, cien metros para hospitales chicos y más de 100 metros — cada vez en progresión geométrica - para hospitales grandes, leyes y reglas establecidas por las mejores autoridades científicas en la materia ${ }^{47}$.

Dickman explicaba que, si el hospital en cuestión poseía 500 camas, la cantidad de metros cuadrados necesaria era de 50000 . Si el hospital, en cambio, contaba con 1000 camas, el espacio mínimo disponible debería de ser de 230000 metros cuadrados. Para avalar su argumento, sostuvo que su cálculo se basaba en «leyes establecidas por todos los autores científicos y consignadas en los mejores libros de la materia». En especial, Dickman también se refirió al libro La construcción de Hospitales, de Vandelvelde y otros, en su segunda edición de 1912, "citado en la discusión del policlínico, pero mal citado», pues allí se consignaba que «la superficie que necesita cada hospital, con cuadros y tablas que establecen que para un nosocomio de 1500 camas, como es el proyectado del policlínico, se necesita una superficie de 230000 metros cuadrados $\rangle^{48}$. Estas cifras chocaban con las ofrecidas por Cantón, dado que si el policlínico José de San Martín debía tener un mínimo de 1200 camas, ello era incompatible con los escasos 97.000 metros cuadrados estipulados como su espacio ideal.

Es un indicio clave la cita realizada por Dickman de la misma obra mencionada por Eliseo Cantón en 1908, pues pone en evidencia semejanzas y diferencias en los estilos de argumentación de ambos autores. La lectura del diputado socialista destacaba el fracaso de los hospitales monstruo y, por ende, la necesidad de reinterpretar aquellas experiencias fracasadas en función de las decisiones a tomar para el sistema clínico porteño. Así, presentaba de manera distinta algunos de los datos que su rival había utilizado, y mencionaba en forma explícita la reedición del libro, acto que le permitía fundamentar en el terreno del saber médico clínico una lectura de mayor actualidad que la vertida por Cantón. Desde esta óptica, se debería dar forma a un siste-

\footnotetext{
47 Ibíd., pp. 114.

48 Ibíd., pp. 114-115.
} 
ma clínico que respetara las condiciones específicas de la ciudad y, por ende, rechazar el transplante de una tecnología hospitalaria por el mero hecho de ser la última moda en Europa. Para otorgar mayor legitimidad a esta interpretación, Dickman mencionaba la figura de Julio Méndez, quien había dictado - en la sociedad de higiene publica e ingeniería sanitaria - un ciclo de conferencias sobre los modelos hospitalarios vigentes por aquellos años y sus problemáticas, ciclo que criticaba el modelo de hospital central ${ }^{49}$.

En términos generales, el proyecto impulsado por Cantón encarnaba para Dickman un conjunto de males éticos, políticos, económicos y científicos que había que dejar atrás y que, por lo tanto, hacían inconcebible su materialización:

Finalmente, se necesita en esta época de democracia real y efectiva demostrar al país que la nación se encamina por la vía de la modestia, de la sobriedad, de la economía positiva y útil; que la honestidad política debe traer la honestidad mental, porque es nuestra desgracia que las altas esferas docentes y científicas, que deben dar el ejemplo a la juventud, le han mostrado siempre con sus hechos los malos caminos, los rumbos desviados que conducen al éxito fácil y ruidoso, sin inculcarles la labor metódica, modesta y disciplinada que exige la honestidad mental como una condición indiscutible de éxito y de progreso. Charlatanismo científico, charlatanismo político, escándalo financiero, todo se liga y se combina, y todo nos viene como una mala herencia de una época detestable, que hay que borrar de la historia del país. En vísperas de una gran campaña nacional, el parlamento de la nación debe dar un alto ejemplo de honestidad administrativa, derogando esta ley, que es un colazo, como he dicho antes, de una mala época, de una época de escándalo y vergüenza; y todos nosotros debemos formular el propósito firme y consciente en este momento de encrucijada de la historia universal, de cambiar costumbres, de modificar prácticas, de encaminarnos hacia el progreso real y positivo, en que la economía, la técnica, el arte, la ciencia sean expresión honesta de un estado de conciencia colectivo, de un estado de labor fecunda y consciente ${ }^{50}$.

\section{A MODO DE EPÍLOGO}

En el debate por la ley 6026 del policlínico José de San Martín se enfrentaron dos destacados miembros de la «clerecía médica local» con convicciones políticas y médicas polares. Desde una perspectiva política que pone de manifiesto una crisis de crecimiento de la conciencia clínica, ambos médicos re-

\footnotetext{
49 Ibíd., p. 127.

50 Ibíd., p. 128.
} 
afirmaron - al discutirlo - el concepto de medicalización de la población de la ciudad de Buenos Aires a partir de la problematización del eje hospitalescama-salud de la población cristalizado durante la década de 1880. En este sentido, se puede pensar que estamos en un punto de bifurcación de trayectorias posibles y divergentes para la práctica de la clínica médica, momento en que se vuelven a poner en cuestión algunos tópicos, cuyos significados pocos años atrás parecían estabilizados. La controversia alrededor de la materialización de «un gran hospital policlínico» preanuncia el clima de crisis en el seno de la profesión médica porteña, que adquirirá rasgos más explícitos durante las décadas de 1920 y $1930^{51}$.

En este sentido, el estudio del debate por el policlínico pone en evidencia las divergencias en el proceso de construcción de un ethos médico vinculado a los dispositivos materiales, que a su vez presuponen y remiten a formas antitéticas de entender los modos de organización y las jerarquías que estructuran la práctica clínica. Es así que los socialistas percibieron en la intención centralizadora de Cantón la potencial cristalización de una correlación de fuerzas que los perjudicaría. De esta forma, mientras que Cantón, a partir de un acceso privilegiado a las estructuras de poder político y académico, apeló para la fundamentación de su proyecto a algunas autoridades científicas europeas, Dickman puso en práctica un estilo de argumentación característico de los sectores médicos críticos con el cuerpo docente desde fines de los años 1870, especialmente cultivado por el Círculo Médico Argentino. Esta sociedad se caracterizó por destacar el valor de la producción y reinterpretación local en cualquier campo del pensamiento médico y sus miembros solían criticar la obsecuencia inherente al acto de aceptación de autoridades científicas extranjeras en forma acrítica y la tendencia del cuerpo docente a encandilarse con el «éxito fácil y ruidoso» ${ }^{52}$.

En la medida en que para los médicos - en tanto grupo social que ocupaba bancas legislativas y que, por comparación con otros sectores del campo científico, tuvo acceso privilegiado a las arcas del estado - el lugar político de su profesión jugó un papel primario en la definición de su ethos, el debate estudiado también pone de manifiesto la colisión de dos concepciones divergentes acerca del sentido y el uso de los bienes públicos. La posición de Cantón fue afín a una representación cosmopolita de la medicina clínica local y no ocultó su tendencia a la magnificencia - expresión simultánea de una estructura de poder y rasgo de civilización-. En Dickman, si bien no está ausente la refe-

51 Belmartino et al. (1988).

52 SOUZA (2005), p. 127. 
rencia a modelos europeos, la cautela y su postura de austeridad en cuanto al uso de los fondos públicos se vinculó a la aceptación de un panorama hospitalario más bien precario. Así, la discusión alrededor del costo del policlínico muestra que detrás del enfrentamiento de dos cosmovisiones médicas subyacen también percepciones excluyentes del papel del estado y sus necesidades respecto de la clínica local y, por lo tanto, representaciones muy diferentes en cuanto a los vínculos de la clínica local con el contexto médico internacional.

Desde la perspectiva de la legitimación política de las iniciativas científicas, también resulta iluminador de los procesos de construcción de significados en el plano cognitivo el uso de la retórica y la práctica parlamentaria, tales como la organización de comisiones especiales, el manejo de los tiempos institucionales, la movilización de la opinión de la corporación médica. Recíprocamente, el debate estudiado también muestra cómo algunos aspectos cognitivos puestos en cuestión se integran como una dimensión ideológica a los factores políticos y sociales para terminar de cerrar cosmovisiones políticas polares para socialistas y conservadores.

En definitiva y de modo general, puede decirse que toda esta movilización de recursos políticos, éticos y cognitivos por parte de dos grupos antagónicos, a la vez que amplificó creencias, intereses y expectativas en pugna sobre el destino de la práctica clínica local, fue vehículo de una voluntad compartida que, desde la perspectiva histórica, permite definir el espacio común del campo de batalla: la voluntad de legitimar un lugar socio-político e institucional del médico como guardián de la objetividad y del orden experimental

Fecha de recepción: 4 de mayo de 2007.

Fecha de aceptación: 11 de septiembre de 2007. 